\title{
Development of an oncological-multidimensional prognostic index (Onco-MPI) for mortality prediction in older cancer patients
}

\author{
Antonella Brunello ${ }^{1}$ (i) - Andrea Fontana ${ }^{2}$ - Valeria Zafferri ${ }^{1} \cdot$ Francesco Panza $^{2,3}$. \\ Pasquale Fiduccia $^{1} \cdot$ Umberto Basso $^{1} \cdot$ Massimiliano Copetti $^{2} \cdot$ Sara Lonardi $^{1}$ \\ Anna Roma $^{1}$ - Cristina Falci ${ }^{4} \cdot$ Silvio Monfardini ${ }^{5} \cdot$ Alberto Cella $^{6}$ - Alberto Pilotto ${ }^{6,7}$. \\ Vittorina Zagonel ${ }^{1}$
}

Received: 26 August 2015 / Accepted: 23 November 2015 / Published online: 12 January 2016

(C) The Author(s) 2016. This article is published with open access at Springerlink.com

\begin{abstract}
Purpose A multidimensional prognostic index (MPI) based on a comprehensive geriatric assessment (CGA) has been developed and validated in independent cohorts of older patients demonstrating good accuracy in predicting one-year mortality. The aim of this study was to develop a cancer-specific modified MPI (Onco-MPI) for mortality prediction in older cancer patients.

Methods We enrolled 658 new cancer subjects $\geq 70$ years (mean age 77.1 years, 433 females, $65.8 \%$ ) attending oncological outpatient services from September 2004 to June 2011. The Onco-MPI was calculated according to a validated algorithm as a weighted linear combination of the following CGA domains: age, sex, basal and instrumental activities of daily living, Eastern Cooperative Oncology
\end{abstract}

Antonella Brunello

antonella.brunello@ioveneto.it

1 Department of Clinical and Experimental Oncology, Medical Oncology 1, Istituto Oncologico Veneto IOV - IRCCS, Padua, Italy

2 IRCCS Casa Sollievo della Sofferenza, San Giovanni Rotondo, Foggia, Italy

3 Neurodegenerative Disease Unit, Department of Basic Medicine, Neuroscience, and Sense Organs, University of Bari Aldo Moro, Bari, Italy

4 Medical Oncology 2, Istituto Oncologico Veneto IOVIRCCS, Padua, Italy

5 IRCCS Fondazione Don Gnocchi, Milan, Italy

6 Department of OrthoGeriatrics, Rehabilitation and Stabilization, Frailty Area, NR-HS Galliera Hospital, Genoa, Italy

7 Geriatrics Unit, Azienda ULSS 16, S Antonio Hospital, Padua, Italy
Group performance status, mini-mental state examination, body mass index, Cumulative Illness Rating Scale, number of drugs and the presence of caregiver. Cancer sites (breast $46.5 \%$, colorectal $21.3 \%$, lung $6.4 \%$, prostate $5.5 \%$, urinary tract $5.0 \%$, other $15.3 \%$ ) and cancer stages (I $37 \%$, II $22 \%$, III $19 \%$, IV $22 \%$ ) were also included in the model. All-cause mortality was recorded. Three grades of severity of the Onco-MPI score (low risk: $0.0-0.46$, medium risk: 0.47-0.63, high risk: $0.64-1.0$ ) were calculated using RECPAM method. Discriminatory power and calibration were assessed by estimating survival C-indices, along with $95 \%$ confidence interval (CI) and the survival-based HosmerLemeshow (HL) measures.

Results One-year mortality incidence rate was $17.4 \%$. A significant difference in mortality rates was observed in Onco-MPI low risk compared to medium- and high-risk patients ( 2.1 vs. 17.7 vs. $80.8 \%, p<0.0001)$. The discriminatory power of one-year mortality prediction of the OncoMPI was very good (survival C-index $0.87,95 \%$ CI 0.84 0.90 ) with an excellent calibration (HL $p$ value 0.854 ).

Conclusion Onco-MPI appears to be a highly accurate and well-calibrated predictive tool for one-year mortality in older cancer patients that can be useful for clinical decision making in this age group.

Keywords Cancer · Elderly · Prognosis · Mortality · Multidimensional prognostic index (MPI) - Comprehensive geriatric assessment (CGA)

\section{Introduction}

In both Europe and in the USA, the majority of persons who receive a cancer diagnosis are aged 65 years or older (Siegel et al. 2012), and the number of older patients with 
cancer is expected to rise substantially in the next decades (Smith et al. 2009). One of the priorities for both clinicians and researchers is the assessment, treatment planning and evaluation of outcomes of these subjects. However, ongoing enrollment biases with underrepresentation of older individuals in clinical trials of cancer (Talarico et al. 2004; Scher and Hurria 2012) greatly limit that evidence-based clinical decisions be taken in such a population. Particularly, determining life expectancy related to functional status and comorbidity seems to be of utmost importance in that it could help in moving beyond arbitrary age-based cutoffs when making decisions of treating older patients with chemotherapy both in the adjuvant setting and for advanced disease (Gill 2012). Several studies demonstrated that in older subjects, the final prognosis is linked to multimorbidity and multidimensional impairment, i.e., an impairment in the functional, cognitive, nutritional and psychosocial domains (Yourman et al. 2012), that are appropriately explored at their best by using a comprehensive geriatric assessment (CGA) also in older patients with cancer (Caillet et al. 2014). Indeed, while recent guidelines recommend life expectancy inclusion in clinical decisionmaking paths in older age (Pilotto et al. 2015), at present, no validated CGA-based prognostic instruments are available to guide treatment plan in older cancer patients (Baijal and Periyakoil 2014).

Recently, a multidimensional prognostic index (MPI) has been developed and validated on the basis of a standardized CGA (Pilotto et al. 2008), which take into account eight domains related to functional and cognitive status, nutrition, comorbidities, pressure sore risk, number of medications and social status. The MPI has been shown to be an accurate predictor for short- and long-term mortality in patients hospitalized for acute or reactivation of chronic diseases such as community-acquired pneumonia, dementia, congestive heart failure, chronic kidney disease, and other most common disorders leading to death in the elderly (Pilotto et al. 2012a, b, c) as well as in hospitalized patients with cancer (Giantin et al. 2013). The aim of this study was to develop a cancer-specific MPI (Onco-MPI) applicable in the outpatient setting to predict mortality in older patients with different types of cancer, so recognizing heterogeneity in this age group and leading to individualized approaches toward cancer treatment.

\section{Methods}

\section{Study population}

Patients aged $\geq 70$ referred to the Geriatric Oncology Program of the Istituto Oncologico Veneto (IOV) in Padova, Italy, from September 2004 to June 2011 were included.
Patients needed to have a confirmed diagnosis of cancer and a complete CGA recorded in the clinical chart to be included. For all patients, the following variables were collected: age, gender, Eastern Cooperative Oncology Group (ECOG) performance status (Oken et al. 1982), associated diseases and their severity graded according to Cumulative Illness Rating Scale (CIRS) (Linn et al. 1968), present medications, the presence of pain, body mass index (BMI), site and stage of primary cancer, cancer treatment planned and/or received, living status/the presence of caregiver, basal and instrumental activities of daily living (ADL, IADL) (Katz et al. 1970; Lawton and Brody. 1969), minimental state examination (MMSE) (Folstein et al. 1975) and the 15-item Geriatric Depression Scale (GDS) (Satin et al. 2009).

All patients were followed up with clinical visits every 3-6 months with a median follow-up time of 2.5 years and a range of $0.0-8.2$ years. For patients who died during the follow-up, the date of death was obtained from clinical charts when available, and it was collected either from death certificates or by contacting demographic offices when not available. For subjects who did not experience the end point, survival time was censored at the time of the last available follow-up visit.

\section{The oncological-multidimensional prognostic index (Onco-MPI)}

To build the Onco-MPI, a weighted sum of the following domains was computed (raw formula): age, sex, ADL, IADL, ECOG performance status, MMSE, BMI, CIRS, number of drugs, the presence of caregiver, cancer sites and cancer stages. Weights were estimated from a multivariable Cox proportional hazard model, within 1 year of follow-up. Each weighted sum was then normalized into a range that varies from 0 (lowest risk) to 1 (highest risk), subtracting the observed raw minimum value (i.e., -2.371) and then dividing such difference by the observed range (minimum to maximum span, i.e., 8.034). Three grades of Onco-MPI severity were estimated using RECursive Partition and AMalgamation (RECPAM) algorithm. At each partitioning step, the method chooses the best binary split (cutoff) to maximize the difference in the outcome of interest. Discriminatory power was assessed by estimating survival C-indices, along with $95 \%$ confidence interval (CI) (Pencina and D'Agostino 2004), and the survivalbased Hosmer-Lemeshow (HL) measure of calibration (D'Agostino and Nam 2004) was also assessed.

\section{Statistical analysis}

Patients' baseline characteristics were reported as mean \pm standard deviation (SD) or frequencies and 
percentage for continuous and categorical variables, respectively. Medians and ranges were reported for continuous variables. The overall survival was defined as the time between date of first visit and death. Mortality incidence rates were reported as the observed number of events for 100 person-years and were compared using a Poisson model. Time-to-death analyses were performed using univariate and multivariate Cox regression models, within 1 year of follow-up, and results were expressed as hazard ratios (HRs) and $95 \%$ CI. Survival curves were reported according to the Kaplan-Meier method.

All statistical analyses were performed using SAS version 9.3 (SAS Institute, Cary, NC).

\section{Results}

Of all older patients $\geq 70$ years referred for evaluation as new patients to the Geriatric Oncology Program from September 2004 to June 2011, full CGA data were available for 658 patients. Baseline patients' characteristics are shown in Table 1.

After a median follow-up time of 2.5 years (range 0.0 8.2), 105 patients died, with an overall one-year mortality incidence rate of $17.4 \%$. Univariate Cox regressions analysis showed that 11 domains of the CGA were significantly associated with mortality (Table 2).

In detail, one-year mortality risk was associated with increasing age, male sex, lower MMSE, impaired ADL, impaired IADL, number of severe comorbidities according to CIRS, poor ECOG performance status, the presence of caregiver and late-stage cancer, whereas having a breast cancer diagnosis (vs. all other cancers) and higher BMI predicted lower mortality.

As shown in Table 3, the weights used to build the OncoMPI were the regression coefficients (logarithm of the HR) estimated from a multivariate Cox model (Table 4). After the normalization procedure, our score ranged from 0 (low risk) to 1 (high risk).

The Onco-MPI score had a good discriminatory power, yielding a C-statistic of 0.869 (95\% CI 0.841-0.897) and a good calibration measure $(\mathrm{HL} p$ value $=0.854)$. Three risk score categories were estimated for Onco-MPI score using RECPAM method, according to the following cutoffs: 0-0.46 (low risk), 0.47-0.63 (moderate risk) and 0.64-1 (high risk). A significant difference in mortality rates was observed for Onco-MPI low risk compared to medium- and high-risk patients (2.1 vs. 17.7 vs. $80.8 \%$, respectively, $p<0.001$ ). Kaplan-Meier survival curves for one-year mortality risk, according to the three risk score categories (low risk, medium risk and high risk), are shown in Fig. 1.

\section{Discussion}

In the present study, the cancer-specific Onco-MPI appeared to be a highly accurate and well-calibrated prognostic tool for one-year mortality in older cancer patients that can be useful for defining homogeneous prognostic categories and clinical decision making in this age group. Indeed, therapeutic decisions in elderly cancer patients are not fully informed unless heterogeneity of the aging process is taken into account. Actually, some forms of CGA have been successfully used to establish individualized treatment plans of treatment (Caillet et al. 2011) and in defining risk of toxicity from treatments in older cancer patients (Hurria et al. 2011; Extermann et al. 2012). Thus, current clinical guidelines for cancer in older age recommend to implement the CGA methodology (Extermann et al. 2005; Biganzoli et al. 2012; Droz et al. 2010; Pallis et al. 2010) in order to determine the residual biological, psychological and functional capabilities of the older patients, i.e., the grade of frailty (Baijal and Periyakoil 2014; Hamaker et al. 2012), for developing a personalized plan for treatments and interventions. Indeed, whatever the definition and methodology used to evaluate frailty, frail patients have a higher mortality compared to non-frail patients. In a previous study, we showed that frail hospitalized patients, being treated despite poor conditions, had poor outcome (Basso et al. 2008). Furthermore, frail lymphoma patients had same outcome whether they were treated with active modified oncological treatment or palliative care (Tucci et al. 2009). The prognostic evaluation of life expectancy emerges thus as a key factor by which pros and cons of active oncological treatment must be weighted, both in the adjuvant setting and in the metastatic setting. Prognosis could be also fundamental for balancing the harm-benefit and cost-benefit ratios in situations of uncertainty when prescribing highcost drugs or treatments requiring multiple admissions with potential impact on quality of life. In recent years, some prognostic scores have been proposed, but none of these was based on information collected by a standardized CGA (Yourman et al. 2012; Pilotto et al. 2015; Baijal and Periyakoil 2014). The MPI has been previously validated in older hospitalized patients suffering from major diseases, including several types of cancer (Pilotto et al. 2012a, b, c; Giantin et al. 2013), with a significant higher predictive power than other widely used frailty indexes (Bellera et al. 2012). In this cohort from an outpatient setting, we developed a modified MPI that included, compared to the originally MPI, the ECOG performance status (instead of the Exton-Smith scale), the MMSE (instead of the Short Portable Mental Status Questionnaire, SPMSQ) and the BMI (instead of the Mini Nutritional Assessment, MNA) 
Table 1 Baseline patients' characteristics

\begin{tabular}{|c|c|c|}
\hline \multirow{2}{*}{$\frac{\text { Variable }}{\text { No of patients }}$} & \multicolumn{2}{|l|}{ Category } \\
\hline & & 658 \\
\hline \multirow[t]{2}{*}{ Age (years) } & Mean \pm SD & $77.16 \pm 5.11$ \\
\hline & Median (min-max) & $77.00(70.00-96.00)$ \\
\hline \multirow[t]{2}{*}{$\operatorname{Sex}(n, \%)$} & Females & $433(65.81 \%)$ \\
\hline & Males & $225(34.19 \%)$ \\
\hline \multirow[t]{2}{*}{ BMI $\left(\mathrm{kg} / \mathrm{m}^{2}\right)$} & Mean \pm SD & $25.49 \pm 4.21$ \\
\hline & Median (min-max) & $25.22(15.50-47.56)$ \\
\hline \multirow[t]{2}{*}{ ADL } & Mean $\pm \mathrm{SD}$ & $5.71 \pm 0.85$ \\
\hline & Median (min-max) & $6.00(0.00-6.00)$ \\
\hline \multirow[t]{2}{*}{ IADL } & Mean $\pm \mathrm{SD}$ & $6.83 \pm 1.80$ \\
\hline & Median (min-max) & $8.00(0.00-8.00)$ \\
\hline \multirow[t]{2}{*}{ The presence of comorbidity $(n, \%)$} & No & $495(75.23 \%)$ \\
\hline & Yes & $163(24.77 \%)$ \\
\hline \multirow[t]{2}{*}{ Comorbidity index CIRS } & Mean \pm SD & $1.71 \pm 1.29$ \\
\hline & Median (min-max) & $2.00(0.00-7.00)$ \\
\hline \multirow[t]{2}{*}{ No of total comorbidities CIRS } & Mean $\pm \mathrm{SD}$ & $2.72 \pm 1.60$ \\
\hline & Median (min-max) & $3.00(0.00-9.00)$ \\
\hline \multirow[t]{2}{*}{ No of severe comorbidities CIRS } & Mean \pm SD & $0.31 \pm 0.60$ \\
\hline & Median (min-max) & $0.00(0.00-4.00)$ \\
\hline \multirow[t]{2}{*}{$\operatorname{MMSE}(n, \%)$} & $<24$ & $119(18.09 \%)$ \\
\hline & $\geq 24$ & $539(81.91 \%)$ \\
\hline \multirow[t]{2}{*}{ The presence of psychiatric disease $(n, \%)$} & No & $606(92.10 \%)$ \\
\hline & Yes & $52(7.90 \%)$ \\
\hline \multirow[t]{4}{*}{ Cancer stage $(n, \%)$} & I & $246(36.39 \%)$ \\
\hline & II & $144(21.88 \%)$ \\
\hline & III & $121(18.39 \%)$ \\
\hline & IV & $147(22.34 \%)$ \\
\hline \multirow[t]{2}{*}{ Cancer treatment $(n, \%)$} & No & $160(24.32 \%)$ \\
\hline & Yes & $498(75.68 \%)$ \\
\hline \multirow[t]{2}{*}{ No of drugs } & Mean \pm SD & $3.32 \pm 2.43$ \\
\hline & Median (min-max) & $3.00(0.00-13.00)$ \\
\hline \multirow[t]{2}{*}{ ECOG performance status } & Mean $\pm \mathrm{SD}$ & $0.52 \pm 0.72$ \\
\hline & Median (min-max) & $0.00(0.00-4.00)$ \\
\hline \multirow[t]{2}{*}{ Caregiver $(n, \%)$} & No & $193(29.33 \%)$ \\
\hline & Yes & $465(70.67 \%)$ \\
\hline \multirow[t]{2}{*}{ 15-item Geriatric Depression Scale $(n, \%)$} & $\leq 5$ & $497(75.53 \%)$ \\
\hline & $>5$ & $161(24.47 \%)$ \\
\hline \multirow[t]{2}{*}{ Geriatric syndromes $(n, \%)$} & No & $609(92.55 \%)$ \\
\hline & Yes & $49(7.45 \%)$ \\
\hline \multirow[t]{6}{*}{ Tumor site $(n, \%)$} & Breast & $306(46.50 \%)$ \\
\hline & Colorectal & $140(21.28 \%)$ \\
\hline & Lung & $42(6.38 \%)$ \\
\hline & Prostate & $36(5.47 \%)$ \\
\hline & Other genitourinary & $33(5.02 \%)$ \\
\hline & Other & $101(15.35 \%)$ \\
\hline
\end{tabular}

$B M I$ body mass index, $A D L$ activities of daily living, IADL instrumental activities of daily living, CIRS Cumulative Illness Rating Scale, MMSE mini-mental state examination, ECOG Eastern Cooperative Oncology Group 
Table 2 Results from univariable Cox regressions for mortality risk prediction, within 1 year of follow-up in older cancer patients

\begin{tabular}{|c|c|c|c|}
\hline Variable & Category & $\mathrm{HR}(95 \% \mathrm{CI})$ & $p$ value \\
\hline Age & Cont. Var. & $1.076(1.039-1.114)$ & $<0.001$ \\
\hline Sex & Male versus female & $2.084(1.422-3.056)$ & $<0.001$ \\
\hline BMI & Cont. Var. & $0.870(0.824-0.918)$ & $<0.001$ \\
\hline $\mathrm{ADL}$ & Cont. Var. & $0.667(0.582-0.764)$ & $<0.001$ \\
\hline IADL & Cont. Var. & $0.765(0.709-0.825)$ & $<0.001$ \\
\hline The presence of comorbidity & Yes versus no & $1.420(0.941-2.145)$ & 0.095 \\
\hline Comorbidity Index CIRS & Cont. Var. & $1.011(0.873-1.173)$ & 0.880 \\
\hline No of total comorbidites CIRS & Cont. Var. & $1.036(0.921-1.164)$ & 0.557 \\
\hline No of severe comorbidites CIRS & Cont. Var. & $1.288(0.985-1.685)$ & 0.065 \\
\hline MMSE & $<24$ versus $\geq 24$ & $1.913(1.253-2.922)$ & 0.003 \\
\hline Psychiatric diseases & Yes versus no & $0.809(0.376-1.742)$ & 0.589 \\
\hline \multirow[t]{3}{*}{ Cancer stage } & IV versus I & $17.089(8.189-35.659)$ & $<0.001$ \\
\hline & III versus I & $4.555(1.966-10.555)$ & $<0.001$ \\
\hline & II versus I & $3.614(1.546-8.443)$ & 0.003 \\
\hline Cancer treatment & Yes versus no & $0.726(0.478-1.105)$ & 0.135 \\
\hline No of drugs & Cont. Var. & $1.036(0.960-1.117)$ & 0.363 \\
\hline Performance status (continuous) & Cont. Var. & $2.133(1.766-2.576)$ & $<0.001$ \\
\hline \multirow[t]{3}{*}{ ECOG performance status (categorical) } & $\geq 3$ versus 0 & $6.184(2.163-17.679)$ & $<0.001$ \\
\hline & 2 versus 0 & $7.418(4.223-13.031)$ & $<0.001$ \\
\hline & 1 versus 0 & $4.232(2.658-6.738)$ & $<0.001$ \\
\hline Caregiver & Yes versus no & $1.603(1.002-2.565)$ & 0.049 \\
\hline 15-item Geriatric Depression Scale & $>5$ versus $\leq 5$ & $1.402(0.926-2.125)$ & 0.111 \\
\hline Syndromes & Yes versus no & $1.329(0.693-2.550)$ & 0.392 \\
\hline \multirow[t]{5}{*}{ Tumor site } & Breast versus other & $0.078(0.038-0.157)$ & $<0.001$ \\
\hline & Colorectal versus other & $0.401(0.234-0.685)$ & $<0.001$ \\
\hline & Lung versus other & $1.429(0.815-2.506)$ & 0.213 \\
\hline & Prostate versus other & $0.335(0.131-0.856)$ & 0.022 \\
\hline & Other genitourinary versus other & $1.372(0.747-2.520)$ & 0.308 \\
\hline
\end{tabular}

$B M I$ body mass index, $A D L$ activities of daily living, IADL instrumental activities of daily living, CIRS Cumulative Illness Rating Scale, $M M S E$ mini-mental state examination, ECOG Eastern Cooperative Oncology Group, Cont. Var. continuous variable

to evaluate functional, cognitive and nutritional status, respectively.

Notably, we further included in the prognostic model both patients' cancer site and cancer stage variables, along with the main MPI domains, due to their clinical relevance on the mortality risk prediction. For tumor sites, some biases can have been introduced since breast cancer was the prevalent type $(46.5 \%)$ with early stages of disease being more represented for this tumor site, whereas other tumor sites, i.e., lung cancer, were far less prevalent, with higher stages of disease at first access. Globally, $75 \%$ of patients received active oncology treatment whose impact on survival was not significant. Therefore, considering multidimensional aggregate information may go beyond the heterogeneity of the sample related to diverse cancer sites and stages, and the variety of treatments used (endocrine agents, several types of chemotherapy regimens).
While screening tools based on abbreviated CGA showed high sensitivity with low specificity in predicting mortality (Smets et al. 2014; Bellera et al. 2012), CGAbased prognostic scores similar to the Onco-MPI in older cancer patients have been poorly investigated. Recently, a study conducted on 249 older Asian cancer patients by using CGA items to build a prognostic nomogram based on six clinical laboratory items demonstrated a relatively low predictive accuracy for one-, two- and three-year overall survival with a C-index value of 0.71 (Kanesvaran et al. 2011). The Onco-MPI was developed on more than 600 patients using some CGA-based items which can be easily implemented in routine oncological practice to drive treatment decisions. A time horizon of 1 year was chosen because we focused on a short-term mortality risk prediction, which is particularly helpful to give systemic oncological treatment or not in many cancers of the older people. 
Table 3 Estimated domains weights used to compute the onco-multidimensional prognostic index (MPI), for mortality risk prediction within 1 year of follow-up

\begin{tabular}{|c|c|c|c|}
\hline \multicolumn{2}{|c|}{ Domains $\left(\mathrm{D}_{\mathbf{i}}\right)$} & Category & $\begin{array}{l}\text { Weights for } \\
\text { onco-MPI }\left(\mathrm{S}_{\mathrm{i}}\right)\end{array}$ \\
\hline \multicolumn{2}{|l|}{ Age (years) } & Cont. Var. & 0.04730 \\
\hline \multicolumn{2}{|l|}{ Sex } & $\begin{array}{l}\text { Female (ref) } \\
\text { Male }\end{array}$ & \begin{tabular}{|l}
0 \\
0.01706 \\
\end{tabular} \\
\hline \multicolumn{2}{|l|}{ BMI } & Cont. Var. & -0.09782 \\
\hline \multicolumn{2}{|l|}{ ADL } & Cont. Var. & -0.07717 \\
\hline \multicolumn{2}{|l|}{ IADL } & Cont. Var. & 0.04983 \\
\hline \multicolumn{2}{|c|}{ ECOG Performance Status } & Cont. Var. & 0.70607 \\
\hline \multicolumn{2}{|c|}{$\mathrm{N}^{\circ}$ of severe comorbidites CIRS } & Cont. Var. & -0.12960 \\
\hline \multicolumn{2}{|l|}{ Cancer stage } & \begin{tabular}{|l|} 
I (ref) \\
II \\
III \\
IV \\
\end{tabular} & \begin{tabular}{|l|}
0 \\
1.11712 \\
0.74957 \\
1.80828 \\
\end{tabular} \\
\hline \multicolumn{2}{|l|}{ Tumour site } & $\begin{array}{l}\text { Other (ref) } \\
\text { Breast } \\
\text { Colorectal } \\
\text { Lung } \\
\text { Prostate } \\
\text { Other genitourinary }\end{array}$ & \begin{tabular}{|l|}
0 \\
-1.93081 \\
-1.03025 \\
0.36265 \\
-1.57998 \\
0.19956 \\
\end{tabular} \\
\hline \multicolumn{2}{|l|}{ MMSE } & Cont. Var. & -0.06270 \\
\hline \multicolumn{2}{|l|}{$\mathrm{N}^{\circ}$ of drugs } & Cont. Var. & -0.01218 \\
\hline \multicolumn{2}{|l|}{ Caregiver } & \begin{tabular}{|l|} 
No $($ ref) \\
Yes \\
\end{tabular} & \begin{tabular}{|l|l} 
\\
0.21035 \\
\end{tabular} \\
\hline \multicolumn{2}{|c|}{ Raw onco-MPI } & $\mathrm{R}=\sum\left(\mathrm{S}_{\mathrm{i}} \mathrm{D}_{\mathrm{i}}\right)$ & \\
\hline \multicolumn{2}{|c|}{$\begin{array}{l}\text { Normalization formula for } \\
\text { onco-MPI }\end{array}$} & $\frac{(R+2.371)}{8.034}$ & \\
\hline \multirow{3}{*}{$\begin{array}{l}\text { Cut-offs } \\
\text { (RECPAM) }\end{array}$} & Low & $0-0.46$ & \\
\hline & Medium & $0.47-0.63$ & \\
\hline & High & $0.64-1.00$ & \\
\hline \multicolumn{2}{|c|}{$\begin{array}{l}\text { Survival c-index } \\
(95 \% \mathrm{CI})^{*}\end{array}$} & $\begin{array}{l}\text { HL test } \\
\text { (p-value)* }\end{array}$ & \\
\hline \multicolumn{2}{|c|}{$0.869(0.841-0.897)$} & 0.854 & \\
\hline
\end{tabular}

Defined using: age, sex, $B M I$ body mass index, $A D L$ activities of daily living, $I A D L$ instrumental activities of daily living, ECOG Eastern Cooperative Oncology Group performance status, CIRS Cumulative Illness Rating Scale, MMSE mini-mental state examination, number of drugs, the presence of a caregiver, cancer stage and tumor size Cont. Var. continuous variable

* Survival C-index, along with $95 \%$ confidence intervals (CI), and $p$ value from Hosmer-Lemeshow (HL) goodness-of-fit test for calibration of the Onco-MPI score within 1 year of follow-up

In this study, ECOG performance status was significantly associated with mortality, thus confirming the wellknown prognostic role of this tool in oncology. Moreover, both ADL and IADL in our model were significantly associated with mortality in univariable analysis, and the loss of significance in the multivariable analysis probably was related to interaction with other considered domains (Extermann et al. 1998). The presence and number of severe comorbidities have also been found to be related to poor prognosis in the present study. Indeed, comorbidity has been consistently proven to be associated with worse survival in older cancer patients (Piccirillo et al. 2004). This has been shown to be independent of ECOG performance status (Firat et al. 2002) and functional status (Extermann et al. 1998). Weight loss or a low BMI was associated by reverse epidemiology with an increased risk of mortality in older age (Landi et al. 2000), a finding confirmed also in our cohort of cancer patients Furthermore, cognitive status showed a strong prognostic value in our older cancer patients, confirming earlier findings suggesting dementia as an independent prognostic factor for survival in older subjects (Wolfson et al. 2001). Depression has been also proposed as a predictive factor of mortality in older cancer patients with controversial findings (Satin et al. 2009). In our study, the presence of depression was not associated with survival, and therefore it was not considered among variables for building the Onco-MPI. In both the geriatric and oncology literature, social isolation has been linked to an increased risk of mortality (Kroenke et al. 2006). In our model, the presence of a caregiver was unexpectedly related to worse survival, probably because in an outpatient setting, those requiring a caregiver could have been the most vulnerable ones.

The calculation of Onco-MPI can be easily performed through an excel file in which the value of each domain (age, sex, BMI, ADL, IADL, ECOG PS, MMSE, number of severe comorbidity, cancer stage, tumor site, number of drugs, the presence of caregiver) is multiplied by the coefficient which is the weight for Onco-MPI (reported in Table 3).

From a practical standpoint, we can take the example of an 80-year-old woman with stage III colorectal cancer, PS 1 , ADL 6/6, IADL 5/8, BMI 28, good cognitive status (MMSE $\geq 24$ ), two severe comorbidity, five drugs, with caregiver present; this patient has an Onco-MPI score of 0.44 , which means that she is in the lower Onco-MPI score, with an estimated risk of mortality at 1 year of $2.1 \%$. The same woman, but having a bad cognitive status (MMSE < 24) and BMI 19, has an Onco-MPI score of 0.54 , which means that she is in the intermediate risk group, with an estimated one-year mortality of $17.7 \%$. If this patient had ADL 5/6, her Onco-MPI would be 0.72 , which corresponds to higher risk of mortality at 1 year, estimated to be more than $80 \%$. While adjuvant treatment may be discussed in the first case and carefully evaluated in the second case, in the third case geriatric assessment and oncoMPI suggest not to consider adjuvant chemotherapy. It is important to notice that the patient was judged as having a PS 1 in all cases, thus confirming that comprehensive geriatric assessment adds information, and in the case of the Onco-MPI adds prognostic information, to simple PS estimation (Repetto et al. 2002).

The main limitations of the present study were the possible selection of more "fit" older cancer patients and the lack of data of the predictive role of the Onco-MPI for 
Table 4 Results from multivariable Cox regressions for mortality risk prediction, within 1 year of follow-up in older cancer patients

\begin{tabular}{|c|c|c|c|}
\hline Variable & Category & $\operatorname{HR}(95 \% \mathrm{CI})$ & $p$ value \\
\hline Age & Cont. Var. & $1.040(0.996-1.086)$ & 0.075 \\
\hline Sex & Male versus female & $1.018(0.647-1.600)$ & 0.939 \\
\hline BMI & Cont. Var. & $0.912(0.859-0.968)$ & 0.002 \\
\hline ADL & Cont. Var. & $0.920(0.751-1.128)$ & 0.423 \\
\hline IADL & Cont. Var. & $1.008(0.870-1.167)$ & 0.92 \\
\hline Comorbidity index CIRS & Cont. Var. & $0.914(0.749-1.114)$ & 0.372 \\
\hline MMSE & $<24$ versus $\geq 24$ & $0.971(0.595-1.584)$ & 0.906 \\
\hline Psychiatric diseases & Yes versus no & $0.498(0.198-1.251)$ & 0.138 \\
\hline \multirow[t]{3}{*}{ Cancer stage } & IV versus I & $6.689(2.950-15.166)$ & $<0.001$ \\
\hline & III versus I & $2.129(0.854-5.306)$ & 0.105 \\
\hline & II versus I & $3.335(1.389-8.009)$ & 0.007 \\
\hline Cancer treatment & Yes versus no & $0.984(0.618-1.567)$ & 0.945 \\
\hline No of drugs & Cont. Var. & $1.023(0.923-1.133)$ & 0.668 \\
\hline \multirow[t]{3}{*}{ ECOG performance status } & $\geq 3$ versus 0 & $7.747(1.744-34.411)$ & 0.007 \\
\hline & 2 versus 0 & $3.827(1.777-8.244)$ & $<0.001$ \\
\hline & 1 versus 0 & $3.122(1.877-5.191)$ & $<0.001$ \\
\hline Caregiver & Yes versus no & $1.193(0.708-2.010)$ & 0.508 \\
\hline 15-item Geriatric Depression Scale & $>5$ versus $\leq 5$ & $0.947(0.574-1.564)$ & 0.833 \\
\hline Syndromes & Yes versus no & $1.050(0.494-2.234)$ & 0.898 \\
\hline \multirow[t]{5}{*}{ Tumor site } & Breast versus other & $0.164(0.068-0.396)$ & $<0.001$ \\
\hline & Colorectal versus other & $0.379(0.214-0.671)$ & $<0.001$ \\
\hline & Lung versus other & $1.407(0.767-2.579)$ & 0.270 \\
\hline & Prostate versus other & $0.209(0.073-0.599)$ & 0.004 \\
\hline & Other genitourinary versus other & $1.260(0.652-2.433)$ & 0.492 \\
\hline
\end{tabular}

$B M I$ body mass index, $A D L$ activities of daily living, IADL instrumental activities of daily living, CIRS Cumulative Illness Rating Scale, MMSE mini-mental state examination, ECOG Eastern Cooperative Oncology Group, Cont. Var. continuous variable

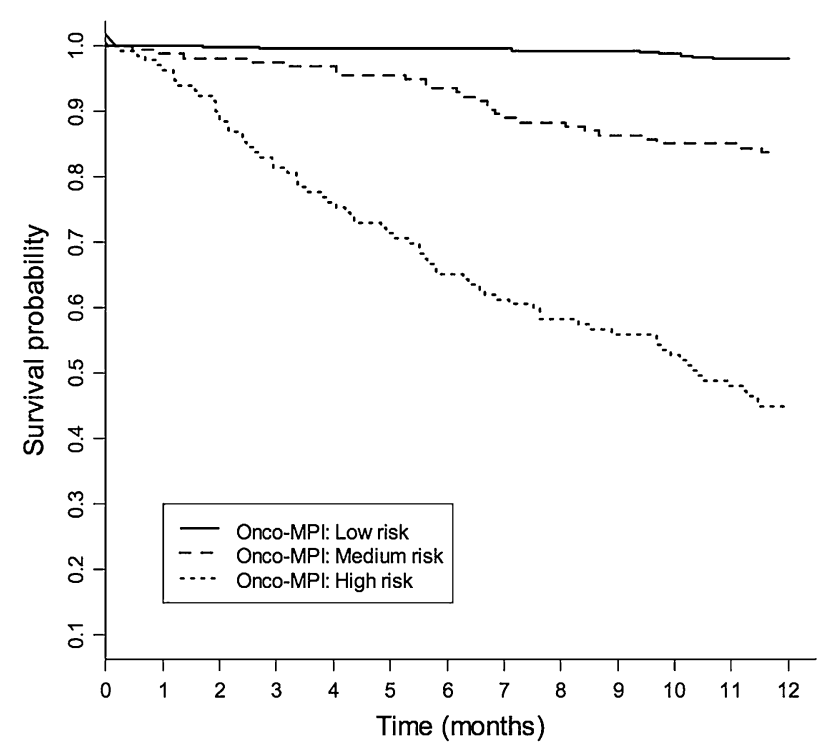

Fig. 1 Kaplan-Meier survival curves, within 1 year of follow-up, according to the three Onco-MPI risk score categories (low risk, medium risk and high risk) mid- and long-term mortality. The Onco-MPI has been built on outpatients; therefore, there is a greater likelihood of the inclusion of less frail patients which may have skewed the results. However, it holds a very good discriminatory power with a C-statistic of 0.87 . In fact, despite the lack of accepted criteria to assess the quality of prognostic indices, generally C-statistics for discrimination can be considered good for ranges $0.70-0.79$, very good for ranges $0.80-0.89$, and excellent for 0.90 or greater (Yourman et al. 2012). Beyond its prognostic ability, the Onco-MPI could also serve as an useful tool for evaluating effectiveness of an intervention in different settings, when changes in Onco-MPI categories that can be obtained may reflect the outcome of the intervention, as recently reported in older patients with late-life major depressive disorder responders and non-responders to antidepressant treatment (Pilotto et al. 2012a, b, c). Finally, the Onco-MPI in the research setting may help to properly classify patients enrolled in clinical trials, selecting more homogeneous subgroups of patients. However, the Onco-MPI warrants external validation, which is 
already underway, and proof of ability to predict mortality with longer follow-up.

Acknowledgments This work was supported by Italian Ministero della Salute (Grant \#GR-2009-1606663) and Regione del Veneto (Ricerca Sanitaria Finalizzata Grant \#317/10). This work was presented as a poster at the 2013 ASCO Annual Meeting [J Clin Oncol 31, 2013 (suppl; abstr 6593)].

\section{Compliance with ethical standards}

Conflict of interest The authors have declared that no competing interests exist.

Ethical approval All procedures performed were in accordance with the ethical standards of the national regulations and with the $1964 \mathrm{Hel}-$ sinki Declaration and its later amendments. The study was approved by IOV Ethics Committee.

Informed consent Informed consent was obtained from all individual participants included in the study.

Open Access This article is distributed under the terms of the Creative Commons Attribution 4.0 International License (http://creativecommons.org/licenses/by/4.0/), which permits unrestricted use, distribution, and reproduction in any medium, provided you give appropriate credit to the original author(s) and the source, provide a link to the Creative Commons license, and indicate if changes were made.

\section{References}

Baijal P, Periyakoil V (2014) Understanding frailty in cancer patients. Cancer J 20(5):358-366

Basso U, Tonti S, Bassi C, Brunello A, Pasetto LM, Scaglione D et al (2008) Management of frail and not-frail elderly cancer patients in a hospital-based geriatric oncology program. Crit Rev Oncol Hematol 66(2):163-170

Bellera CA, Rainfray M, Mathoulin-Pélissier S, Mertens C, Delva F, Fonck M et al (2012) Screening older cancer patients: first evaluation of the G-8 geriatric screening tool. Ann Oncol 23(8):2166-2172

Biganzoli L, Wildiers H, Oakman C, Marotti L, Loibl S, Kunkler I et al (2012) Management of elderly patients with breast cancer: updated recommendations of the International Society of Geriatric Oncology (SIOG) and European Society of Breast Cancer Specialists (EUSOMA). Lancet Oncol 13(4):e148-e160

Caillet P, Canoui-Poitrine F, Vouriot J, Berle M, Reinald N, Krypciak $S$ et al (2011) Comprehensive geriatric assessment in the decision-making process in elderly patients with cancer: ELCAPA study. J Clin Oncol 29(27):3636-3642

Caillet P, Laurent M, Bastuji-Garin S, Liuu E, Culine S, Lagrange JL, Canoui-Poitrine F, Paillaud E (2014) Optimal management of elderly cancer patients: usefulness of the comprehensive geriatric assessment. Clin Interv Aging 29(9):1645-1660

D'Agostino RB, Nam BH (2004) Evaluation of the performance of survival analysis models: discrimination and calibration measures. In: Balakrishnan N, Rao CR (eds) Handbook of statistics. Elsevier BV, Amsterdam, pp 1-25

Droz JP, Balducci L, Bolla M, Emberton M, Fitzpatrick JM, Joniau S et al (2010) Management of prostate cancer in older men: recommendations of a working group of the International Society of Geriatric Oncology. BJU Int 106(4):462-469
Extermann M, Overcash J, Lyman GH, Parr J, Balducci L (1998) Comorbidity and functional status are independent in older cancer patients. J Clin Oncol 16(4):1582-1587

Extermann M, AaproM Bernabei R, Cohen HJ, Droz JP, Lichtman S et al (2005) Use of comprehensive geriatric assessment in older cancer patients: recommendations from the task force on CGA of the International Society of Geriatric Oncology (SIOG). Crit Rev Oncol Hematol 55(3):241-252

Extermann M, Boler I, Reich RR, Lyman GH, Brown RH, DeFelice J et al (2012) Predicting the risk of chemotherapy toxicity in older patients: the chemotherapy risk assessment scale for high-age patients (CRASH) score. Cancer 118(13):3377-3386

Firat S, Bousamra M, Gore E, Byhardt RW (2002) Comorbidity and KPS are independent prognostic factors in stage I non-small-cell lung cancer. Int J Radiat Oncol Biol Phys 52(4):1047-1057

Folstein MF, Folstein SE, McHugh PR (1975) "Mini-mental state". A practical method for grading the cognitive state of patients for the clinician. J Psychiatr Res 12(3):189-198

Giantin V, Valentini E, Iasevoli M, Falci C, Siviero P, De Luca E et al (2013) Does the multidimensional prognostic index (MPI), based on a comprehensive geriatric assessment (CGA), predict mortality in cancer patients? Results of a prospective observational trial. J Geriatr Oncol 4(3):208-217

Gill TM (2012) The central role of prognosis in clinical decision making. JAMA 307(2):199-200

Hamaker ME, Jonker JM, de Rooij SE, Vos AG, Smorenburg CH, van Munster BC (2012) Frailty screening methods for predicting outcome of a comprehensive geriatric assessment in elderly patients with cancer: a systematic review. Lancet Oncol 13(10): e437-e444

Hurria A, Togawa K, Mohile SG, Owusu C, Klepin HD, Gross CP et al (2011) Predicting chemotherapy toxicity in older adults with cancer: a prospective multicenter study. J Clin Oncol 29(25):3457-3465

Kanesvaran R, Li H, Koo KN, Poon D (2011) Analysis of prognostic factors of comprehensive geriatric assessment and development of a clinical scoring system in elderly Asian patients with cancer. J Clin Oncol 29(27):3620-3627

Katz S, Downs TD, Cash HR, Grotz RC (1970) Progress in the development of an index of ADL. Gerontologist 10(1):20-30

Kroenke CH, Kubzansky LD, Schernhammer ES, Holmes MD, Kawachi I (2006) Social networks, social support, and survival after breast cancer diagnosis. J Clin Oncol 24(7):1105-1111

Landi F, Onder G, Gambassi G, Pedone C, Carbonin P, Bernabei R (2000) Body mass index and mortality among hospitalized patients. Arch Intern Med 160(17):2641-2644

Lawton MP, Brody EM (1969) Assessment of older people: selfmaintaining and instrumental activities of daily living. Gerontologist 9(3):179-186

Linn B, Linn M, Gurel L (1968) The cumulative illness rating scale. J Am Geriatr Soc 16(5):622-626

Oken MM, Creech RH, Tormey DC, Horton J, Davis TE, McFadden ET et al (1982) Toxicity and response criteria of the Eastern Cooperative Oncology Group. Am J Clin Oncol 5:649-655

Pallis AG, Gridelli C, van Meerbeeck JP, Greillier L, Wedding U, Lacombe D et al (2010) EORTC Elderly Task Force and Lung Cancer Group and International Society for Geriatric Oncology (SIOG) experts' opinion for the treatment of non-small-cell lung cancer in an elderly population. Ann Oncol 21(4):692-706

Pencina MJ, D'Agostino RB (2004) Overall C as a measure of discrimination in survival analysis: model specific population value and confidence interval estimation. Stat Med 23(13):2109-2123

Piccirillo JF, Tierney RM, Costas I, Grove L, Spitznagel EL Jr (2004) Prognostic importance of comorbidity in a hospital-based cancer registry. JAMA 291(20):2441-2447 
Pilotto A, Ferrucci L, Franceschi M, D’Ambrosio LP, Scarcelli C, Cascavilla L et al (2008) Development and validation of a multidimensional prognostic index for one-year mortality from comprehensive geriatric assessment in hospitalized older patients. Rejuvenation Res 11(1):151-161

Pilotto A, D'Onofrio G, Panza F, Copetti M, Cascavilla L, Paris F et al (2012a) Treatment of late-life major depressive disorder with selective serotonin reuptake inhibitors improves the multidimensional prognostic index. J Clin Psychopharmacol 32(5):726-729

Pilotto A, Panza F, Ferrucci L (2012b) A multidimensional prognostic index in common conditions leading to death in older patients. Arch Intern Med 172(7):594-595

Pilotto A, Rengo F, Marchionni N, Sancarlo D, Fontana A, Panza F et al (2012c) FIRI-SIGG Study Group. Comparing the prognostic accuracy for all-cause mortality of frailty instruments: a multicentre 1-year follow-up in hospitalized older patients. PLoS ONE 7(1):e29090

Pilotto A, Sancarlo D, Daragjati J, Panza F (2015) Perspective: the challenge of clinical decision-making for drug treatment in older people. The role of multidimensional assessment and prognosis. Front Med 1:61

Repetto L, Fratino L, Audisio RA, Venturino A, Gianni W, Vercelli M et al (2002) Comprehensive geriatric assessment adds information to Eastern Cooperative Oncology Group performance status in elderly cancer patients: an Italian Group for Geriatric Oncology Study. J Clin Oncol 20(2):494-502

Satin JR, Linden W, Phillips MJ (2009) Depression as a predictor of disease progression and mortality in cancer patients: a metaanalysis. Cancer 115(22):5349-5361
Scher KS, Hurria A (2012) Under-representation of older adults in cancer registration trials: known problem, little progress. J Clin Oncol 30(17):2036-2038

Siegel R, Naishadham D, Jemal A (2012) Cancer statistics. CA Cancer J Clin 62(1): 10-29

Smets IH, Kempen GI, Janssen-Heijnen ML, Deckx L, Buntinx FJ, van den Akker M (2014) Four screening instruments for frailty in older patients with and without cancer. BMC Geriatr 26(14):26

Smith BD, Smith GL, Hurria A, Hortobagyi GN, Buchholz TA (2009) Future of cancer incidence in the United States: burdens upon an aging, changing nation. J Clin Oncol 27(17):2758-2765

Talarico L, Chen G, Pazdur R (2004) Enrollment of elderly patients in clinical trials for cancer drug registration: a 7-year experience by the US Food and Drug Administration. J Clin Oncol 22(22):4626-4631

Tucci A, Ferrari S, Bottelli C, Borlenghi E, Drera M, Rossi G (2009) Comprehensive geriatric assessment is more effective than clinical judgment to identify elderly diffuse large cell lymphoma patients who benefit from aggressive therapy. Cancer 115(19):4547-4553

Wolfson C, Wolfson DB, Asgharian M, M'Lan CE, Ostbye T, Rockwood $\mathrm{K}$ et al (2001) A reevaluation of the duration of survival after the onset of dementia. N Engl J Med 344(15):1111-1116

Yourman LC, Lee SJ, Schonberg MA, Widera EW, Smith AK (2012) Prognostic indices for older adults. A systematic review. JAMA 307(2):182-192 